\title{
Do Merging Dwarf Galaxies Explain a Peculiar Gravitational-Wave Detection?
}

\author{
The hard-to-explain masses of two coalescing black holes could be \\ accounted for if they were the central black holes in two distant, tiny \\ galaxies that merged.
}

By Rachel Berkowitz

( n May 21, 2019, the LIGO and Virgo collaborations recorded an unexpected gravitational-wave detection. The signal, designated GW190521, emanated from the coalescence of two black holes whose masses fall into a range that's forbidden by conventional stellar evolution theories (see Viewpoint: A Heavyweight Merger). Now, Antonella Palmese, at Fermi National Accelerator Laboratory (Fermilab) and the University of Chicago, and Christopher Conselice, at the University of Manchester, UK, propose that the observation can be explained by the merger of the black holes at the centers of two low-mass dwarf galaxies [1]. Such "forbidden mass" black holes could form in dwarf galaxies from the successive coalescence of multiple smaller black holes-without needing to rewrite models of stellar black hole formation.

To test their proposal, the researchers first extrapolated the

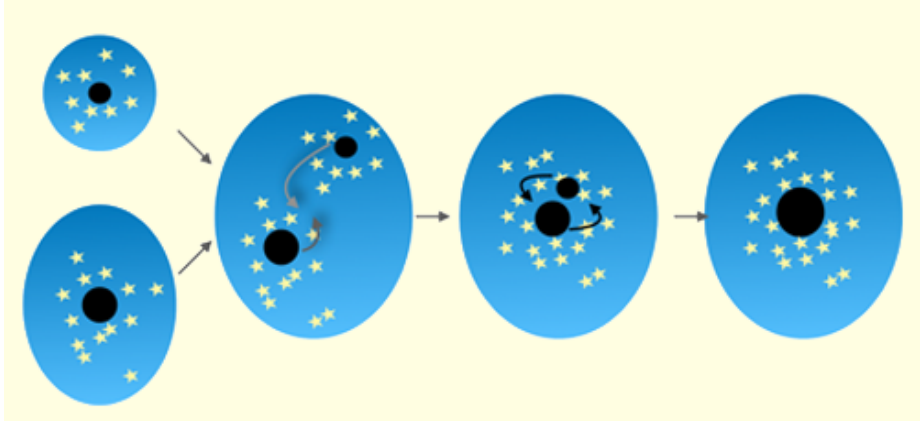

masses of black holes at the centers of dwarf galaxies based on known relationships between the central black hole mass and the total stellar mass for larger galaxies. Then, using observational datasets detailing how the population of dwarf galaxies has changed over time, they calculated the merger rate of these galaxies as a function of their distance from Earth. Finally, they determined how long it should take for two galaxies' central black holes to coalesce after the galaxies themselves had begun to merge.

By estimating how many dwarf galaxies are likely to host black holes similar in mass to those suspected for GW190521, Palmese and Conselice concluded that a dwarf galaxy merger is a plausible explanation. Observations of other mergers involving black holes in this mass range, and further numerical analyses of black holes in nearby galaxies and clusters, could confirm the scenario and open a new avenue to understanding galaxy formation and the still-mysterious origin of supermassive black holes.

Rachel Berkowitz is a Corresponding Editor for Physics based in vancouver, Canada.

\section{REFERENCES}

1. A. Palmese and C. J. Conselice, "GW190521 from the merger of ultradwarf galaxies,” Phys. Rev. Lett. 126, 181103 (2021).

Credit: A. Palmese/Fermilab 